\title{
Dabigatran Versus Warfarin After Bioprosthesis Valve Replacement for the Management of Atrial Fibrillation Postoperatively: DAWA Pilot Study
}

\author{
André Rodrigues Durães ${ }^{1}$ Pollianna de Souza Roriz ${ }^{1} \cdot$ Bianca de Almeida Nunes $^{1}$. \\ Felipe Pinho e Albuquerque ${ }^{1}$ - Fábio Vieira de Bulhões ${ }^{1} \cdot$ Andre Mauricio de Souza $^{2}$ \\ Fernandes $^{1} \cdot$ Roque Aras ${ }^{1}$
}

Published online: 18 February 2016

(c) The Author(s) 2016. This article is published with open access at Springerlink.com

\begin{abstract}
Objectives Dabigatran is a direct thrombin inhibitor shown to be an effective alternative to warfarin in patients with non-valvular atrial fibrillation (AF). We evaluated the use of dabigatran in patients with bioprosthetic mitral and/ or aortic valve replacement and $\mathrm{AF}$.

Methods We selected 34 and randomized 27 patients in a 1:1 ratio to receive dabigatran or warfarin. The primary endpoint was the presence of a new intracardiac thrombus at 90 days, by transesophageal echocardiogram (TEE). Secondary endpoints included the development of dense spontaneous echo contrast (SEC) and incidence of stroke (ischemic or hemorrhagic), myocardium infarction, valve thrombosis and peripheral embolic events.

Results The trial was terminated prematurely because of low enrollment. There were 27 patients in total: 15 patients placed in the dabigatran group and 12 in the warfarin
\end{abstract}

André Rodrigues Durães

andreduraes@gmail.com

Pollianna de Souza Roriz

polliroriz@yahoo.com.br

Bianca de Almeida Nunes

bianunes@gmail.com

Felipe Pinho e Albuquerque

pinho66@hotmail.com

Fábio Vieira de Bulhões

fvbulhoes@yahoo.com.br

Andre Mauricio de Souza Fernandes

andremsf@hotmail.com

Roque Aras

roque.aras@uol.com.br

1 Hospital Ana Nery/UFBa, Rua Saldanha Marinho, S/N, Caixa D́Agua, Salvador, Brazil group. After 90 days, one patient $(8.3 \%)$ in the warfarin group and none in the dabigatran group had developed a new intracardiac thrombus. In the dabigatran group, two patients $(13.3 \%)$ developed dense SEC versus one patient $(8.3 \%)$ in the warfarin group. In the warfarin group, one patient $(8.3 \%)$ presented ischemic stroke, and none did in the dabigatran group. We observed no cases of hemorrhagic stroke, valve thrombosis, embolic events or myocardial infarction in either group throughout the study. However, one patient $(6.7 \%)$ in the dabigatran group had a fully recovered transient ischemic attack and one patient in the warfarin group died of heart failure.

Conclusions The use of dabigatran appears to be similar to warfarin in preventing the formation of intracardiac thrombus.

Trial Registration Clinicaltrials.gov NCT01868243.

\section{Key Points}

There are no published study in humans evaluating the efficacy and safety of dabigatran or any other NOACs in patients with mitral and/or aortic bioprosthesis valve.

DAWA is a phase 2, prospective, open-label, randomized, pilot study. The main variable to be observed in this study is intracardiac thrombus. There are no formal primary or secondary clinical efficacy or safety outcomes because it is a pilot study.

The DAWA study encourages a larger multicentric prospective study to assess the use of new oral anticoagulants in patients with bioprosthesis valve. 


\section{Introduction}

Thromboembolism and anticoagulant-related bleeding represent the majority of complications experienced by prosthetic valve recipients. It is estimated that 4 million valve replacement procedures have been performed in the last 50 years and it remains the only definitive treatment for most patients with advanced heart valve disease [1]. Oral anticoagulation with warfarin and similar vitamin $\mathrm{K}$ antagonists (VKAs) is recommended lifelong for patients with bioprosthesis who have other indications for anticoagulation, such as atrial fibrillation (AF) [2]. Even with the appropriate use of therapy, there is a high incidence of thromboembolic events: $1-4 \%$ per year. Furthermore, bleeding risk is significant, ranging from 2 to $9 \%$ per year. Because of VKAs' narrow therapeutic index, interactions, genetic variants, and need for blood monitoring of patients, different anticoagulants with more predictable pharmacological effects have been searched for. Alternatives to warfarin are now available [3].

Dabigatran etexilate is the prodrug of dabigatran, a direct thrombin (factor IIa) inhibitor, which was approved by the US Food and Drug Administration (FDA) for the prevention of stroke and systemic embolism in patients with nonvalvular AF, and has also been approved for the treatment of acute deep vein thrombosis and pulmonary embolism. Antithrombotic therapy for thromboprophylaxis in patients with mechanical heart valves remains challenging $[4,5]$. Until now, the Oral Dabigatran Etexilate in Patients after Heart Valve Replacement (RE-ALIGN) trial [6] comparing dabigatran etexilate to warfarin was the only randomized controlled study in patients with mechanical valve prosthesis, but it was terminated prematurely because of an excess of thromboembolic and bleeding events among patients in the dabigatran group. Additional studies are needed to find suitable alternatives to VKAs in this population [7]. There are no published studies in humans evaluating the efficacy and safety of dabigatran or any other new oral anticoagulant (NOAC) in patients with mitral and/or aortic bioprosthesis valve. The Dabigatran Versus Warfarin After Bioprosthesis Valve Replacement for the Management of Atrial Fibrillation Postoperatively (DAWA) study was designed to provide the first clinical trial that tested dabigatran use in patients with bioprosthesis and AF postoperatively.

\section{Methods}

\subsection{Study Design and Oversight}

DAWA is a phase II, prospective, open-label, randomized, pilot study. The main variable to be observed in this study is intracardiac thrombus. There are no formal primary or secondary clinical efficacy or safety outcomes because it is a pilot study. Mortality and morbidity events (reversible ischemic neurological deficit, ischemic and hemorrhagic stroke, systemic embolism, any bleeding, prosthesis valve thrombosis and death) were evaluated in an exploratory manner. The details of the trial design have been previously described [8]. The trial protocol was approved by the local ethics and research committee in the city of SalvadorBrazil (under protocol number 14284813.9.0000.0045), and written informed consent was obtained from all patients. An independent data and safety monitoring board closely monitored the trial. All the authors contributed to the interpretation of the results, wrote the first version of the manuscript and approved all versions, made the decision to submit the manuscript for publication, and vouch for the accuracy and completeness of the data reported and the fidelity of this article to the study protocol.

\subsection{Patients and Randomization}

Patients eligible for inclusion in the study were 18-64 years old, underwent mitral and/or aortic bioprosthesis valve replacement at least 3 months prior to entering the study and had documented AF postoperatively in addition to exclusion of atrial thrombus or valve prosthesis thrombosis by transesophageal echocardiogram (TEE). Non-contrast brain computed tomography (CT) without hemorrhage or findings of acute cerebral infarction on the last 2 days of screening was also necessary. The complete list of inclusion and exclusion criteria is provided in the protocol [8].

Patients were randomly assigned to receive dabigatran or warfarin by a computer generated list of random numbers performed to a 1:1 ratio between the groups. Following that, the allocation sequence was concealed from the researcher enrolling participants in sequentially numbered, opaque, black, sealed envelopes. After randomization, patients had study visits scheduled at 7 days (via telephone) and at 30 days (personally), with a monthly follow-up for 90 days. After this, non-contrast brain CT (or magnetic resonance imaging when necessary) and TEE were repeated in all randomized patients. The former was executed to document possible cerebral events with no clinical expression and the latter to analyze the incidence of intracardiac thrombi, new dense spontaneous echo contrast (SEC) or its resolution, in addition to thrombosis or dysfunction of valvular prosthesis.

\subsection{Drug Administration Protocol}

Patients assigned to the dabigatran group received $110 \mathrm{mg}$ of dabigatran etexilate twice daily. Patients with previous 
use of warfarin underwent washout with immediate introduction of dabigatran once the international normalized ratio (INR) was $<2.5$. The warfarin dose was adjusted to maintain the INR between 2.0 and 3.0. Doses were between 5 and $10 \mathrm{mg}$ in the first days for most individuals, with subsequent dosing based on INR response.

\subsection{Outcomes}

The primary endpoint was the detection of intracardiac thrombus in TEE at the end of follow-up (90 days). Additional efficacy and safety outcomes included dense SEC, stroke (ischemic or hemorrhagic), reversible ischemic neurological deficit, systemic embolism, prosthesis valve thrombosis, bleeding event (major or minor), elevated liver enzymes or hepatic function abnormalities and death.

TEE was performed using a commercially available ultrasound imaging system (iE33; Philips Medical Systems, Andover, MA, USA) with a 3-dimensional matrix-array transesophageal transducer. Left atrial (LA) abnormalities such as thrombus and dense SEC were assessed by TEE in all patients. Dense SEC was defined as a dynamic smokelike signal that swirled slowly in a circular pattern within the LA and appendage, with a gradation of $>2+[9,10]$.

The bleeding risk was based on the criteria of the Control of Anticoagulation Subcommittee of the International Society on Thrombosis and Haemostasis [11] and HAS-BLED (Hypertension, Abnormal Renal/Liver Function, Stroke, Bleeding History or Predisposition, Labile International Normalized Ratio, Elderly, Drugs/Alcohol) score [12].

\subsection{Statistical Considerations}

The SPSS 17.0 (SPSS Inc., Chicago, IL, USA) was used to perform statistical analysis of the collected data. The primary analysis was performed according to the intent-totreat principle. A safety analysis was performed on all patients treated, regardless of any protocol violations. Quantitative variables were described as mean and standard deviation. The mean comparison was performed by the Student $t$ test for independent populations or related populations, as appropriate. The qualitative and categorical variables were presented as percentages, and their comparisons were made by the Fisher exact test.

\section{Results}

\subsection{Study Discontinuation}

The data and safety monitoring board recommended discontinuation of the study on September 1, 2014 because of a significant drop in recruitment. All participating patients discontinued the assigned study drug and were switched to warfarin.

\subsection{Patients and Follow-Up}

A total of 34 patients were selected between August 2013 and November 2014 (six were excluded for previous intracardiac thrombus; one for unstable INR control). Of the 27 randomized, 15 were assigned to receive dabigatran and 12 to receive warfarin. The characteristics of the patients at baseline were well balanced (Table 1). The majority of patients were female (66.7 and $58.3 \%$ ), young adults (mean $48.8 \pm 10.4$ and $45.7 \pm 6$; median 45 and 44.5) who had undergone isolated mitral valve replacement (73.3 and $75 \%$ ), with few risk factors in both groups (hypertension 46.7 and $50 \%$; diabetes 7.1 and $0 \%$; smoking 13.3 and $25 \%$; previous stroke 26.7 and $33.3 \%$ ), and with low-risk surgery (logist euroSCORE mean $1.6 \% \pm 0.4$ and $1.9 \% \pm 1.5$ ), in the dabigatran and warfarin groups, respectively (Table 1). One patient did not finish the follow-up because he/she died. All others made use of the randomized drug by the scheduled period (up of 90 days).

\subsection{Clinical Outcomes}

Intracardiac thrombus occurred in one patient $(8.3 \%)$ in the warfarin group. One case $(8.3 \%)$ of ischemic stroke occurred in the warfarin group, and one case $(6.7 \%)$ of reversible ischemic neurological deficit was observed in the dabigatran group. Bleeding occurred in one patient in the dabigatran group $(6.7 \%)$ and two patients $(16.7 \%)$ in the warfarin group, respectively. One case of hospitalization in each group was seen, without statistical significance (Table 2).

Dense SEC was detected in seven patients $(46.7 \%)$ in the dabigatran group and three patients $(25 \%)$ in the warfarin group [hazard ratio $0.38 ; 95 \%$ confidence interval (CI) $0.10-2.00 ; P=0.23$ ] at the end of the study. Resolution and new SEC occurred in one $(6.7 \%)$ versus one patient $(8.3 \%)$ (hazard ratio $1.30 ; 95 \%$ CI $0.10-22$; $P=0.70)$ and two $(13.3 \%)$ versus one patient $(8.3 \%)$ (hazard ratio $0.60 ; 95 \% \mathrm{CI} 0.10-7.4 ; P=0.58$ ) in the dabigatran and warfarin groups, respectively (Table 3 ).

\section{Discussion}

Utilizing the good accuracy of TEE for detecting LA thrombi and brain CT scans in the detection of ischemic and hemorrhagic cerebrovascular events, we compared dabigatran $110 \mathrm{mg}$ twice daily with adjusted-dose warfarin, 
Table 1 Baseline characteristics

\begin{tabular}{lll}
\hline & Dabigatran $(n=15)$ & Warfarin $(n=12)$ \\
\hline Male, no. (\%) & $5(33.3)$ & $5(41.7)$ \\
Age (years) & & \\
Mean & $48.8 \pm 10.4$ & $45.7 \pm 6$ \\
Median & 45 & 44.5 \\
Range & $37-67$ & $37-54$ \\
Hypertension, no. (\%) & $7(46.7)$ & $6(50)$ \\
Diabetes, no. (\%) & $1(7.1)$ & 0 \\
Smoking, no. $(\%)^{\mathrm{a}}$ & $2(13.3)$ & $3(25)$ \\
Previous stroke & $4(26.7)$ & $4(33.3)$ \\
Isolated mitral replacement & $11(73.3)$ & $9(75)$ \\
LVEF, mean (\%) & $40 \pm 12$ & $50 \pm 10$ \\
NYHA (III-IV), no. (\%) & $5(33.3)$ & $3(27.3)$ \\
Logistic euroSCORE II, mean $(\%)^{\mathrm{b}}$ & $1.6 \pm 0.4$ & $1.9 \pm 1.5$ \\
Left atrium, mean (mm) & $58 \pm 10$ & $53 \pm 13$ \\
HAS-BLED ${ }^{\mathrm{c}}$, median & $0(0-1)$ & $0(0-1)$ \\
\hline
\end{tabular}

Plus-minus values are means \pm SD. No significant differences were noted between the groups

$H A S-B L E D$ hypertension, abnormal renal/liver function, stroke, bleeding history or predisposition, labile international normalized ratio, elderly, drugs/alcohol, LVEF left ventricular ejection fraction, NYHA New York Heart Association SD standard deviation

${ }^{\text {a }}$ Previous or actual

b The logistic European System for Cardiac Operative Risk Evaluation (euroSCORE), which measures risk at the time of cardiovascular surgery, is calculated with the use of a logistic-regression equation. A score of $>20$ indicates a very high surgical risk

c A score of $\geq 3$ suggests increased bleeding risk and warrants some caution and/or regular review

Table 2 Efficacy and safety outcomes, according to treatment group

\begin{tabular}{|c|c|c|c|c|}
\hline Event & Dabigatran (no. of patients) & Warfarin (no. of patients) & $\begin{array}{l}\text { Relative risk } \\
\text { (95\% } \\
\text { confidence } \\
\text { interval) }\end{array}$ & $P$ value \\
\hline Intracardiac thrombus & 0 & $1(8.3)$ & $1.1(0.9-1.3)$ & 0.42 \\
\hline Stroke or systemic embolism & 0 & $1(8.3)$ & $1.1(0.9-1.3)$ & 0.44 \\
\hline Reversible ischemic neurological deficit & $1(6.7)$ & 0 & $0.9(0.8-1.0)$ & 0.55 \\
\hline Bleeding $^{\mathrm{a}}$ & $1(6.7)$ & $2(16.7)$ & $2.8(0.2-35)$ & 0.41 \\
\hline Hospitalization & $1(6.7)$ & $1(8.3)$ & $1.3(0.7-22)$ & 0.70 \\
\hline Death & 0 & $1(8.3)$ & $1.1(0.9-1.3)$ & 0.44 \\
\hline
\end{tabular}

Values are number $(\%)$ unless indicated otherwise

$H A S$ - $B L E D$ hypertension, abnormal renal/liver function, stroke, bleeding history or predisposition, labile international normalized ratio, elderly, drugs/alcohol, $N A$ not applicable

a According criteria of Control of Anticoagulation Subcommittee of the International Society on Thrombosis and Haemostasis and HAS-BLED score

administered in an unblinded fashion, in patients at least 3 months after bioprosthesis replacement and with AF postoperatively, having as the main goal the detection of intracardiac thrombus. However, the trial was stopped early because of a significant decrease of eligible candidates for recruitment. Among the most important reasons for this, we detected a high rate of intracardiac thrombus in the selection phase, low socioeconomic status in many others, and addition to the negative results of the Re-align study.

Despite the small sample size of this randomized pilot study, to the best of our knowledge, our current study is the first that has held a direct comparison between an NOAC (dabigatran) and warfarin in patients with a bioprosthesis valve and AF until now. The primary and all secondary endpoints had quantitatively few events in both groups. 
Table 3 Spontaneous echo contrast and TTR analysis

\begin{tabular}{|c|c|c|c|c|}
\hline Event & Dabigatran (no. of patients) & Warfarin (no of patients) & $\begin{array}{l}\text { Relative risk } \\
\text { (95\% } \\
\text { confidence } \\
\text { interval) }\end{array}$ & $P$ value \\
\hline \multicolumn{5}{|l|}{ Dense SEC } \\
\hline At baseline & $6(40)$ & $3(25)$ & $0.50(0.1-2.6)$ & 0.34 \\
\hline At the end & $7(46.7)$ & $3(25)$ & $0.38(0.1-2.0)$ & 0.23 \\
\hline Resolution of SEC ${ }^{\mathrm{a}}$ & $1(6.7)$ & $1(8.3)$ & $1.30(0.1-22)$ & 0.70 \\
\hline New SEC ${ }^{b}$ & $2(13.3)$ & $1(8.3)$ & $0.60(0.1-7.4)$ & 0.58 \\
\hline Mean TTR (\%) & NA & $66.5 \pm 7$ & NA & NA \\
\hline \multicolumn{5}{|c|}{ Values are number $(\%)$ unless indicated otherwise } \\
\hline \multicolumn{5}{|c|}{$\begin{array}{l}\text { NA not applicable, } S E C \text { spontaneous echo contrast, TEE transesophageal echocardiogram, TTR time in } \\
\text { therapeutic range }\end{array}$} \\
\hline
\end{tabular}

The bioprosthesis emerged last century with the expectation of replacing existing mechanical prosthesis, due to not theoretically requiring permanent oral anticoagulation. Actually, the recommendations of the main international guidelines on antithrombotic therapy after bioprosthesis implantation demonstrate a low level of evidence (Grade C), which may be explained by the lack of randomized trials in this scenario [13].

However, certain patients with bioprosthesis may require long-term anticoagulant therapy when there are other indications, in particular with AF. In these cases, thrombogenicity is due to AF and not to the prosthesis. It can be expected that the current absence of indication of NOACs for mechanical prosthesis will be an incentive to continue, or even strengthen, the trend towards favoring implantations of bioprosthesis at the expense of mechanical valves [14].

The reason for evaluating the formation of thrombus or SEC by TEE is justified since they are independent predictors for thromboembolic events, as evidenced in several previous studies. About $30-60 \%$ of patients with SEC or intracardiac thrombus evolve with major thromboembolic events [15]. Patients with AF and dense SEC have a high likelihood of cerebral embolism (22\%) and/or death, despite oral anticoagulation [16]. Furthermore, microembolization of small thrombi derived from the fibrillating LA may be significant causes of silent brain infarction in nonvalvular AF patients [9].

Regarding the dosage of dabigatran $110 \mathrm{mg}$ twice a day chosen in this study, it was based on the results of the Long-term Multicenter Extension of Dabigatran Treatment in Patients with Atrial Fibrillation (RELY-ABLE) trial, which showed no significant difference in stroke or mortality, but a higher rate of major bleeding with the higher versus lower dabigatran dose (150 vs. $110 \mathrm{mg}$ twice a day, respectively) and no difference between the doses in net clinical benefit as estimated by the composite of stroke, bleeding and death [17]. Recently, a separate analysis of the two low-dose NOAC regimens (which included dabigatran) showed that although they have a similar efficacy to warfarin for protection against all stroke or systemic embolic events, they are not as effective for protection against ischemic stroke in particular. However, they do have a safer profile than warfarin and preserve the mortality benefit noted with the high-dose regimens [18].

Beyond that, it is possible that patients with $\mathrm{AF}$ and bioprosthesis or valve repair have a risk of thromboembolism not substantially different from that with more common forms of 'non-valvular' $\mathrm{AF}$, and in any case, on the basis of preliminary evidence accrued from trials with NOACs, there is no evidence of different efficacy or safety compared with warfarin [19].

About neurological events, in the RE-ALIGN study [6], it is important to see that most thromboembolic events among patients in the dabigatran group occurred in "population A" (patients who had started a study drug within 7 days after valve surgery), with fewer occurring in "population B" (patients who had undergone valve implantation more than 3 months before randomization). Besides, stroke, death and major bleeding occurred only in the first group. All patients in our study have more than a 3-month interval from surgery for recruitment, a period known to have a lower incidence of embolic events [20].

There are several limitations of DAWA, among which we highlight the following: unicentric pilot study; small sample size; and short follow-up (90 days) for the occurrence of major clinical events. Despite this, it can be very useful as a hypothesis generator for a large randomized trial.

In summary, the DAWA study encourages a larger multicentric prospective study to assess the use of this NOAC in such a population (bioprosthesis) since usual 
doses would be enough to avoid the formation of thrombi and its several complications, including hard endpoints such as prosthesis thrombosis, stroke or major bleeding.

\section{Compliance with Ethical Standards}

We appreciate the efforts of the institution Ana Nery Hospital. Special thanks to Dr. Edmundo José Nassri Câmara, Dr. Pedro Henrique de Araujo Machado, Dr. Erenaldo Junior, Italvar Neto and Juliana Muniz. This research received no grant from any funding agency in the public, commercial or not-for-profit sectors.

Author André Rodrigues Durães has no conflict of interest to declare. Author Pollianna de Souza Roriz has no conflict of interest to declare. Author Bianca de Almeida Nunes has no conflict of interest to declare. Author Felipe Pinho e Albuquerque has no conflict of interest to declare. Author Fábio Vieira de Bulhões has no conflict of interest to declare. Author Andre Mauricio de Souza Fernandes has no conflict of interest to declare. Author Roque Aras has no conflict of interest to declare.

Open Access This article is distributed under the terms of the Creative Commons Attribution-NonCommercial 4.0 International License (http://creativecommons.org/licenses/by-nc/4.0/), which permits any noncommercial use, distribution, and reproduction in any medium, provided you give appropriate credit to the original author(s) and the source, provide a link to the Creative Commons license, and indicate if changes were made.

\section{References}

1. Sun JC, Davidson MJ, Lamy A, Eikelboom JW. Antithrombotic management of patients with prosthetic heart valves: current evidence and future trends. Lancet. 2009;374(9689): $565-76$.

2. Vahanian A, Alfieri O, Andreotti F, et al. Guidelines on the management of valvular heart disease (version 2012): the Joint Task Force on the Management of Valvular Heart Disease of the European Society of Cardiology (ESC) and the European Association for Cardio-Thoracic Surgery (EACTS). Eur J Cardiothorac Surg. 2012;42(4):S1-44.

3. Mega JL, Simon T. Pharmacology of antithrombotic drugs: an assessment of oral antiplatelet and anticoagulant treatments. Lancet. 2015;S0140-6736(15):60243-4.

4. O'Dell KM, Igawa D, Hsin J. New oral anticoagulants for atrial fibrillation: a review of clinical trials. Clin Ther. 2012;34(4):894-901.

5. Akwaa F, Spyropoulos AC. The potential of target-specific oral anticoagulants for the acute and long-term treatment of venous thromboembolism. Curr Med Res Opin. 2014;30(11):2179-90.
6. Eikelboom JW, Connolly SJ, Brueckmann M, et al. Dabigatran versus warfarin in patients with mechanical heart valves. N Engl J Med. 2013;369(13):1206-14.

7. Forsberg P, DeSancho MT. Role of novel anticoagulants for patients with mechanical heart valves. Curr Atheroscler Rep. 2014;16(11):448.

8. Duraes AR, Roriz PD, Bulhoes FV, et al. Dabigatran versus warfarin after bioprosthesis valve replacement for the management of atrial fibrillation postoperatively: protocol. JMIR Res Protoc. 2014;3(2):e21.

9. Sugioka K, Takagi M, Sakamoto S, et al. Predictors of silent brain infarction on magnetic resonance imaging in patients with nonvalvular atrial fibrillation: a transesophageal echocardiographic study. Am Heart J. 2015;169(6):783-90.

10. Fatkin D, Kelly RP, Feneley MP. Relations between left atrial appendage blood flow velocity, spontaneous echocardiographic contrast and thromboembolic risk in vivo. J Am Coll Cardiol. 1994;23(4):961-9.

11. Schulman S, Kearon C. Definition of major bleeding in clinical investigations of antihemostatic medicinal products in non-surgical patients. J Thromb Haemost. 2005;3(4):692-4.

12. Pisters R, Lane DA, Nieuwlaat R, et al. A novel user-friendly score (HAS-BLED) to assess 1-year risk of major bleeding in patients with atrial fibrillation: the Euro Heart Survey. Chest. 2010;138(5):1093-100.

13. Duraes AR, Duraes MA, Correia LC, et al. Antithrombotic strategy in the three first months following bioprosthetic heart valve implantation. Arq Bras Cardiol. 2013;101(5):466-72.

14. Iung B, Vahanian A. Lessons from the RE-ALIGN trial. Arch Cardiovasc Dis. 2014;107(5):277-9.

15. Ramos AI, Magalhaes AM, Maldonado M, et al. Incidence of intracardiac thrombus and thromboembolism in the first three months after bioprosthetic valve implantation. Arq Bras.Cardiol. 2004;83 Spec No:46-52.

16. Bernhardt P, Schmidt H, Hammerstingl C, et al. Patients with atrial fibrillation and dense spontaneous echo contrast at high risk: a prospective and serial follow-up over 12 months with transesophageal echocardiography and cerebral magnetic resonance imaging. J Am Coll Cardiol. 2005;45(11):1807-12.

17. Connolly SJ, Wallentin L, Ezekowitz MD. The long-term multicenter observational study of dabigatran treatment in patients with atrial fibrillation (RELY-ABLE) study. Circulation. 2013;128(3):237-43.

18. Ruff CT, Giugliano RP, Braunwald E. Comparison of the efficacy and safety of new oral anticoagulants with warfarin in patients with atrial fibrillation: a meta-analysis of randomised trials. Lancet. 2014;383(9921):955-62.

19. De Caterina R, Camm AJ. What is 'valvular' atrial fibrillation? A reappraisal. Eur Heart J. 2014;35(47):3328-35.

20. Duraes AR, Duraes Milena AO, Correia LCL, et al. Antithrombotic strategy in the three first months following bioprosthetic heart valve implantation. Arq Bras Cardiol. 2013;101(5):466-72. 